\title{
Maternal Effects of Immediate versus Delayed Umbilical Cord Clamping: A Randomized Clinical Trial
}

\author{
Gabriela Françoes Rostirolla ${ }^{1,2}{ }^{*}$, Maria Alexandrina Zanatta ${ }^{3}$, Charles Francisco Ferreira ${ }^{1}$, \\ José Geraldo Lopes Ramos ${ }^{1,3}$, Edimárlei Gonsales Valério', Janete Vettorazzi, ${ }^{1,3}$ \\ ${ }^{1}$ Postgraduation Program in Health Sciences: Gynecology and Obstetrics (PPGGO), Faculdade de Medicina (FAMED), \\ Universidade Federal do Rio Grande do Sul (UFRGS), Porto Alegre, Brasil \\ ${ }^{2}$ Universidade de Caxias do Sul (UCS), Caxias do Sul, Brasil \\ ${ }^{3}$ Gynecology and Obstetrics Department, Clinical Hospital of Porto Alegre (HCPA), Faculdade de Medicina (FAMED), \\ Universidade Federal do Rio Grande do Sul Rio Grande do Sul (UFRGS), Porto Alegre, Brasil \\ Email: `gabirosti@gmail.com, malexandrina.zanatta@gmail.com,charlesferreiraufrgs@gmail.com, jramos@hcpa.edu.br, \\ evalerio@hcpa.edu.br, jvettorazzi@hcpa.edu.br
}

How to cite this paper: Rostirolla, G.F., Zanatta, M.A., Ferreira, C.F., Ramos, J.G.L., Valério, E.G. and Vettorazzi, J. (2021) Maternal Effects of Immediate versus Delayed Umbilical Cord Clamping: A Randomized Clinical Trial. Open Journal of Obstetrics and Gynecology, 11, 1500-1511. https://doi.org/10.4236/ojog.2021.1111141

Received: October 4, 2021

Accepted: November 14, 2021

Published: November 17, 2021

Copyright $\odot 2021$ by author(s) and Scientific Research Publishing Inc. This work is licensed under the Creative Commons Attribution International License (CC BY 4.0).

http://creativecommons.org/licenses/by/4.0/

\begin{abstract}
OBJECTIVE: To compare maternal preoperative and postoperative hemoglobin variation after cord clamping. METHODS: Randomized clinical trial performed in Porto Alegre, RS from January to December 2012. It was included 356 women with habitual risk gestations. In the immediate cord clamping group, the umbilical cord section was realized between 0 and $60 \mathrm{~s}$ (group 1) while in the delayed cord clamping group it was realized $>60 \mathrm{~s}$ (group 2). RESULTS: The mean ( \pm standard deviation [SD]) preoperative hemoglobin was $12.13 \pm 1.06$ in the group 1 and $12.13 \pm 1.11$ in the group 2 . The mean $( \pm \mathrm{SD})$ postoperative day 2 hemoglobin level was $10.19 \pm 1.46$ in the group 1 and $10.24 \pm 1.42$ in the group 2. CONCLUSIONS: Delayed umbilical cord clamping resulted in a similar maternal hemoglobin level reduction at postoperative day 2 .
\end{abstract}

\section{Keywords}

Cord Clamping, Delayed Cord Clamping, Immediate Cord Clamping, Matern Hemoglobin Level

\section{Introduction}

The third period of childbirth is characterized by the moment after birth until placental expulsion. The physiological evolution of this period is given without 
routine use of uterotonics, with cord clamping being performed after cessation of pulse and expulsion of the placenta by maternal effort [1] [2]. The active management of the third period of delivery is performed with routine use of 10 units of intramuscular oxytocin after detachment of the newborn's shoulders, followed by clamping and immediate sectioning of the umbilical cord and controlled traction of the placenta, immediate umbilical cord clamping is no longer recommended, except in cases where the newborn needs urgent care, with no absolute contraindication for delayed umbilical cord clamping (between 1 and 3 min after birth, for term newborns). This practice is recommended in the third period of delivery as a way to promote maternal and newborn health [3] [4] [5].

In relation to the benefits of the delayed umbilical cord clamping, the American College of Obstetricians and Gynecologists in January 2017 expanded the recommendation for delayed umbilical cord clamping by at least $30-60 \mathrm{~s}$ after birth for premature newborns to both preterm and term infants; the American Heart Association and the American Academy of Pediatrics recommend umbilical cord clamping between 60 and $180 \mathrm{~s}$ [6] [7] [8]. Although evidence for neonatal benefits with delayed umbilical cord clamping is strong, data related to maternal outcomes are lacking, especially after cesarean section [9].

The high rate of maternal deaths, especially in the poorest and developing countries, has been the subject of numerous public health discussions and strategies aimed at reducing these numbers. The administration of oxytocin to all parturients shortly after shoulder removal, immediate umbilical cord clamping and moderate traction for placental removal is recommended measures to reduce maternal mortality [2] [5]. A review comparing active and expectant management of the third period concluded that active management significantly reduces bleeding $( \pm 1000 \mathrm{~mL})$ in the general population, reducing by $60 \%$ the incidence of postpartum hemorrhage caused by uterine atony and the need for blood products and other complications [10]. The routine recommendation is that all parturients receive 10 units of intramuscular or intravenous oxytocin during the third period of delivery and in cesarean section, as it is the first choice uterotonic for postpartum hemorrhage prophylaxis [11]. In the immediate postpartum period, uterine atony is responsible for $80 \%$ of bleeding cases, totaling 14 million cases per year and occurring in 1 out of every 20 births. Preventing puerperal hemorrhage with active management of the third period is an important strategy in reducing maternal morbidity and mortality [12].

Delayed umbilical cord clamping has clear benefits for the neonate [9]. However, the possible repercussions for maternal health are not clear, justifying a study to evaluate its outcomes. Only one study in the literature has assessed maternal mortality and hemorrhage as a primary outcome, and further studies are needed to assess maternal and neonatal outcomes after the change in active management of the third period of delivery. In this sense, we conducted a non-blinded, randomized controlled trial comparing delayed versus immediate umbilical cord clamping effects in maternal hematimetric parameters (hemoglobin and hematocrit levels) and the occurrence of maternal and newborn post- 
partum complications.

\section{Methods}

\subsection{Trial Design and Study Participants}

This interventional, non-blinded randomized controlled trial involved two groups of parturients admitted to the Clinical Hospital of Porto Alegre (HCPA), Porto Alegre, RS, Brazil, carried out between January and December 2012. The first group had the ligature and umbilical cord section immediately after birth (named immediate umbilical cord clamping group, defined as clamping <60 s after birth in the term conceptuses). The second group had the ligature and umbilical cord section between 60 and $180 \mathrm{~s}$ after birth (named delayed umbilical cord clamping group) in term conceptuses with evident vitality at birth.

\subsection{Outcome Measures}

The primary outcome was maternal hemoglobin variation before (preoperative) and $48 \mathrm{~h}$ after (postoperative) delivery with immediate and delayed umbilical cord clamping. The secondary outcomes evaluated the effects of umbilical cord clamping procedure on maternal hematimetric parameters, the occurrence of maternal (e.g., uterine hypotonia, bleeding, laceration) and neonatal (e.g., neonatal intensive care unit, transient tachypnea of the newborn) complications, as well as the days of hospital discharge.

\subsection{Sample Size, Selection and Randomization}

Winpepi ${ }^{\circledR}$ software, version 11.43 , was used to calculate the sample size [13]. Considering the hypothesis that delayed umbilical cord clamping leads to a decrease of $0.3 \mathrm{~g} / \mathrm{dL}$ in maternal hemoglobin in relation to immediate umbilical cord clamping, a power of $80 \%$, and an alpha error of $0.05,148$ participants were necessary (74 in the immediate and 74 in the delayed umbilical cord clamping groups). Women with a habitual risk pregnancy were included. Women with any of the following conditions were excluded: gestational age $<37$ weeks, HIV positive, $\mathrm{Rh}$ isoimmunization, severe maternal anemia (hemoglobin $<9 \mathrm{~g} / \mathrm{dL}$ ), gestational diabetes, placenta previa, placenta detachment, hemolysis, elevated liver enzymes, HELLP syndrome (hemolysis, elevated liver enzymes, low platelet count), or maternal coagulopathy. Women were also excluded if their newborns presented cord-round-the-neck, hypotonia, or meconium in amniotic fluid. A statistician (who did not participate in the project) randomly allocated the participants to receive immediate or delayed umbilical cord clamping, using a computer-generated randomization sequence (randomization.com), which generated the randomization table. Random number codes were placed in envelopes sealed with the participant's sequence number on the outside of the envelope.

\subsection{Data Organization and Analyses}

Regarding the data processing, the database double entry and review were per- 
formed using the SPSS, version 18.0. (SPSS Inc., Released 2009, PASW Statistics for Windows, Version 18.0. Chicago). Symmetrical quantitative data are expressed as mean \pm standard deviation (SD) or standard error of mean (SEM), or by median and interquartile range $\left(25^{\text {th }}\right.$ and $75^{\text {th }}$ percentiles [P25 - P75]). Categorical variables are described as absolute $(\mathrm{n})$ and relative $(\mathrm{n} \%)$ frequencies. To compare means between groups, Student's $t$-test for independent samples was applied. In asymmetry cases, the Mann-Whitney $U$ test was used to perform comparisons. Associations between categorical variables were conducted by the chi square test with adjusted residual analysis. Spearman's correlations were applied between variables of interest. Significance was set at $5 \%$ for all analyses.

\subsection{Ethics}

Before the collection of any data, ethical approval was obtained from the institutional review board of HCPA (Group of Research and Postgraduation of the Hospital de Clínicas de Porto Alegre, Ref. No. 11.0524). The study was conducted in accordance with the 1964 Declaration of Helsinki and its later amendments of comparable ethical standards (as revised in 2000). All participants gave their informed consent before participation, and all information about the participants was kept strictly confidential. This research did not involve physical, moral, or psychological harm to the participants. Additionally, the trial was registered with Brazilian Registry of Clinical Trials (ReBec, Ref. No. UTN: U1111-1233-9573).

\section{Results}

Of the 3815 births in 2012, 3459 were not included in the study considering the exclusion criteria (meconium, $\mathrm{n}=180$; hypotonic newborn, $\mathrm{n}=180$; circular umbilical cord, $n=726$; indication by pediatrician, $n=311$; prematurity $n=294$; maternal diseases [anemia, diabetes mellitus, HIV, arterial hypertension, and coagulopathies], $\mathrm{n}=557$; placental change, $\mathrm{n}=76$; and non-elective cesarian section, $n=692$ ) or declined to participate/other reasons, for example, in the night shift there was no researcher for data collection $(n=443)$. Maternal and obstetric baseline characteristics of the study participants are shown in Table 1. For the women, the median [P25 - P75] age and body mass index (BMI) were 25.00 [21.00 - 31.00] years and $29.02[26.55-32.05] \mathrm{kg} / \mathrm{m}^{2}$, respectively, and $69.4 \%$ (247/356) were white. The median [P25 - P75] gestational age was 39.57 [38.86 - 40.43] weeks, 195 (54.8\%) gestations were vaginal deliveries, and most of them were performed without episiotomy (51.4\%) and forceps (94.7\%), and they did not use oxytocin (55.1\%), misoprostol (96.1\%), or methylergometrine (94.7\%). The mean $( \pm \mathrm{SD})$ newborn weight was $3304.59 \pm 348.10 \mathrm{~g}$.

Maternal complications are displayed in Table 2. The rates of maternal laceration $(21.3 \%)$, uterine atony $(21.3 \%)$, moderate $(19.8 \%)$ or severe $(0.6 \%)$ bleeding, and maternal median [P25 - P75] days of hospital discharge (2.00 [2.00 3.00] days) were similar between immediate and delayed umbilical cord clamping (chi square or Mann-Whitney $U$ test, $\mathrm{p}>0.05$ for all). The median [P25 - 
P75] additional oxytocin units used was higher in the delayed umbilical cord clamping group $(10.00[10.00-40.00])$ in relation to the immediate umbilical cord clamping group (10.00 [10.00 - 30.00]; Mann-Whitney $U$ test, $\mathrm{p}=0.019)$.

The comparison of average hemoglobin and hematocrit levels from the preoperative to 48-h postoperative times are presented in Table 3. Both umbilical cord clamping groups were similar in all assessed characteristics (Student's

Table 1. Maternal and obstetric characteristics.

\begin{tabular}{|c|c|c|c|c|}
\hline Variable & $\begin{array}{c}\text { Total } \\
(\mathrm{N}=356)\end{array}$ & $\begin{array}{l}\text { Early cord clamping } \\
\qquad(\mathrm{n}=114)\end{array}$ & $\begin{array}{l}\text { Late cord clamping } \\
\qquad(\mathrm{n}=242)\end{array}$ & $\mathrm{P}^{*}$ \\
\hline \multicolumn{5}{|c|}{ Maternal variables } \\
\hline $\begin{array}{l}\text { Age (years): md [P25 - P75] } \\
\text { (minimum - maximum) }\end{array}$ & $\begin{array}{l}25.00[21.00-31.00] \\
\quad(12.00-43.00)\end{array}$ & $\begin{array}{l}26.50[21.00-32.00] \\
\quad(12.00-43.00)\end{array}$ & $\begin{array}{l}25.00[21.00-31.00] \\
\quad(14.00-43.00)\end{array}$ & 0.169 \\
\hline \multicolumn{5}{|l|}{ Ethnicity: n (n\%) } \\
\hline White & $247(69.4)$ & $79(69.3)$ & $168(69.4)$ & 1.000 \\
\hline Not white & $109(30.6)$ & $35(30.7)$ & $74(30.6)$ & \\
\hline $\begin{array}{l}\text { Weight (kg): md [P25 - P75] } \\
\text { (minimum - maximum) }\end{array}$ & $\begin{array}{l}76.00[58.00-86.00] \\
\quad(49.00-151.00)\end{array}$ & $\begin{array}{l}77.00[67.00-88.00] \\
\quad(56.00-151.00)\end{array}$ & $\begin{array}{c}76.00[68.80-86.00] \\
(49.00-135.00)\end{array}$ & 0.851 \\
\hline $\begin{array}{l}\text { Height }(\mathrm{m}) \text { : mean } \pm \mathrm{SD} \\
\quad(\text { minimum - maximum })\end{array}$ & $\begin{array}{c}1.62 \pm 0.06 \\
(1.41-1.80)\end{array}$ & $\begin{array}{c}1.63 \pm 0.06 \\
(1.49-1.78)\end{array}$ & $\begin{array}{c}1.61 \pm 0.07 \\
(1.41-1.80)\end{array}$ & 0.009 \\
\hline $\begin{array}{l}\text { Body mass index }\left(\mathrm{kg} / \mathrm{m}^{2}\right): \text { md }[\mathrm{P} 25-\mathrm{P} 75]^{\mathrm{a}} \\
\quad(\text { minimum - maximum })\end{array}$ & $\begin{array}{l}29.02[26.55-32.05] \\
\quad(19.37-57.54)\end{array}$ & $\begin{array}{l}28.71[25.85-32.00] \\
\quad(19.37-57.54)\end{array}$ & $\begin{array}{l}29.27[27.10-32.05] \\
\quad(20.29-51.44)\end{array}$ & 0.140 \\
\hline \multicolumn{5}{|c|}{ Obstetric variables } \\
\hline $\begin{array}{l}\text { Gestational age (weeks): md [P25 - P75] } \\
\quad(\text { minimum - maximum) }\end{array}$ & $\begin{array}{l}39.57[38.86-40.43] \\
\quad(37.00-43.86)\end{array}$ & $\begin{array}{c}39.57[38.57-40.43] \\
\quad(37.00-41.86)\end{array}$ & $\begin{array}{l}39.57[38.86-40.43] \\
\quad(37.00-43.86)\end{array}$ & 0.628 \\
\hline \multicolumn{5}{|l|}{ Type of delivery: $\mathrm{n}(\mathrm{n} \%)$} \\
\hline Vaginal & $195(54.8)$ & $65(57.0)$ & $130(53.70)$ & 0.570 \\
\hline Cesarean section & $161(45.2)$ & $49(43.0)$ & $112(46.3)$ & \\
\hline \multicolumn{5}{|l|}{ Episiotomy: n (n\%) } \\
\hline No & $183(51.4)$ & $57(50.0)$ & $126(52.1)$ & 0.734 \\
\hline Yes & $173(48.6)$ & $57(50.0)$ & $116(47.9)$ & \\
\hline \multicolumn{5}{|l|}{ Forceps: $\mathrm{n}(\mathrm{n} \%)$} \\
\hline No & $337(94.7)$ & $112(98.2)$ & $225(93.0)$ & 0.070 \\
\hline Yes & $19(5.3)$ & $2(1.8)$ & $17(7.0)$ & \\
\hline \multicolumn{5}{|l|}{ Oxytocin use-(n\%) } \\
\hline No & $196(55.1)$ & $71(62.3)$ & $125(51.7)$ & 0.068 \\
\hline Yes & $160(44.9)$ & $43(37.7)$ & $117(48.3)$ & \\
\hline \multicolumn{5}{|l|}{ Misoprostol use: n (n\%) } \\
\hline No & $342(96.1)$ & $110(96.5)$ & $232(95.9)$ & \\
\hline Yes & $14(3.9)$ & $4(3.5)$ & $10(4.1)$ & 1.000 \\
\hline \multicolumn{5}{|l|}{ Methylergometrine use: n (n\%) } \\
\hline No & $337(94.7)$ & $110(96.5)$ & $227(93.8)$ & 0.423 \\
\hline Yes & $19(5.3)$ & $4(3.5)$ & $15(6.2)$ & \\
\hline
\end{tabular}

Abbreviations: $\mathrm{n}$-absolute frequency; $\mathrm{n} \%$-relative frequency; md-median; P25 - P75-interquartile range $\left(25^{\text {th }}-75^{\text {th }}\right.$ percentiles); SD—standard deviation. ${ }^{a} \mathrm{~N}=355$ : early cord clamping, $\mathrm{n}=114$; late cord clamping, $\mathrm{n}=241 .{ }^{*}$ Chi-Square test with adjusted residual analysis, Student's $t$-test for independent samples, or Mann-Whitney $U$ test. Significance set at $5 \%$ for all analyses. 
Table 2. Maternal complications.

\begin{tabular}{|c|c|c|c|c|}
\hline Variable & $\begin{array}{c}\text { Total } \\
(\mathrm{N}=356)\end{array}$ & $\begin{array}{l}\text { Immediate umbilical } \\
\text { cord clamping }(n=114)\end{array}$ & $\begin{array}{l}\text { Delayed umbilical cord } \\
\text { clamping }(\mathrm{n}=242)\end{array}$ & $\mathrm{P}^{*}$ \\
\hline \multicolumn{5}{|c|}{ Maternal variables } \\
\hline \multicolumn{5}{|l|}{ Laceration: $\mathrm{n}(\mathrm{n} \%)$} \\
\hline No & $280(78.7)$ & $84(73.7)$ & $196(81.0)$ & 0.152 \\
\hline Yes & $76(21.3)$ & $30(26.3)$ & $45(19.0)$ & \\
\hline \multicolumn{5}{|l|}{ Uterine atony: $\mathrm{n}(\mathrm{n} \%)$} \\
\hline No & $280(78.7)$ & $84(73.7)$ & $196(81.0)$ & 0.152 \\
\hline Yes & $76(21.3)$ & $30(26.3)$ & $46(19.0)$ & \\
\hline \multicolumn{5}{|l|}{ Global impression: $\mathrm{n}(\mathrm{n} \%)$} \\
\hline Bleeding & $282(79.7)$ & $91(79.8)$ & $191(79.6)$ & \multirow{3}{*}{0.617} \\
\hline Moderate bleeding & $70(19.8)$ & $23(20.2)$ & $47(19.6)$ & \\
\hline Severe bleeding & $2(0.6)$ & $0(0.0)$ & $2(0.8)$ & \\
\hline \multicolumn{5}{|l|}{ Uterotonic use: $\mathrm{n}(\mathrm{n} \%)$} \\
\hline No & $197(55.3)$ & $71(62.3)$ & $126(52.1)$ & 0.086 \\
\hline Yes & $159(44.7)$ & $43(37.7)$ & $116(47.9)$ & \\
\hline $\begin{array}{l}\text { Oxytocin unit use: md [P25 - P75] } \\
\text { (minimum - maximum) }\end{array}$ & $\begin{array}{l}10.00[10.00-40.00] \\
\quad(10.00-70.00)\end{array}$ & $\begin{array}{l}10.00[10.00-30.00] \\
\quad(10.00-50.00)\end{array}$ & $\begin{array}{l}10.00[10.00-40.00] \\
\quad(10.00-70.00)\end{array}$ & 0.019 \\
\hline $\begin{array}{l}\text { Hospital discharge (days): md [P25 - P75] } \\
\quad \text { (minimum - maximum) }\end{array}$ & $\begin{array}{l}2.00[2.00-3.00] \\
\quad(2.00-22.00)\end{array}$ & $\begin{array}{l}2.00[2.00-3.00] \\
\quad(2.00-6.00)\end{array}$ & $\begin{array}{l}2.00[2.00-3.00] \\
\quad(2.00-22.00)\end{array}$ & 0.398 \\
\hline
\end{tabular}

Abbreviations: $\mathrm{n}$-absolute frequency; $\mathrm{n} \%$-relative frequency; md-median; P25 - P75-interquartile range $\left(25^{\text {th }}-75^{\text {th }}\right.$ percentiles); SD—standard deviation. ${ }^{a} \mathrm{~N}=355$ : early cord clamping, $\mathrm{n}=114$; late cord clamping, $\mathrm{n}=241 .{ }^{*}$ Chi-Square test with adjusted residual analysis, Student's $t$-test for independent samples, or Mann-Whitney $U$ test. Significance set at $5 \%$ for all analyses.

Table 3. Comparison of average hemoglobin and hematocrit levels before and after birth between early and late cord clamping.

\begin{tabular}{|c|c|c|c|c|}
\hline Variable & $\begin{array}{c}\text { Total } \\
(\mathrm{N}=356)\end{array}$ & $\begin{array}{l}\text { Immediate umbilical } \\
\text { cord clamping }(n=114)\end{array}$ & $\begin{array}{l}\text { Delayed umbilical cord } \\
\text { clamping }(n=242)\end{array}$ & $\mathrm{P}^{*}$ \\
\hline \multicolumn{5}{|c|}{ Maternal variables } \\
\hline $\begin{array}{l}\text { Preoperative hematocrit level: mean } \pm \text { SD } \\
\quad(\text { minimum - maximum) }\end{array}$ & $\begin{array}{c}35.59 \pm 3.04 \\
(26.40-45.10)\end{array}$ & $\begin{array}{c}35.73 \pm 2.97 \\
(28.10-43.10)\end{array}$ & $\begin{array}{c}35.52 \pm 3.08 \\
(26.40-45.10)\end{array}$ & 0.535 \\
\hline $\begin{array}{l}\text { Postoperative hematocrit level: mean } \pm \mathrm{SD} \\
\quad(\text { minimum - maximum })\end{array}$ & $\begin{array}{c}30.31 \pm 4.18 \\
(19.00-41.60)\end{array}$ & $\begin{array}{c}30.27 \pm 4.29 \\
(19.50-41.60)\end{array}$ & $\begin{array}{c}30.33 \pm 4.14 \\
(19.00-41.00)\end{array}$ & 0.900 \\
\hline $\begin{array}{l}\text { Preoperative hemoglobin level: mean } \pm \text { SD } \\
\text { (minimum - maximum) }\end{array}$ & $\begin{array}{l}12.13 \pm 1.10 \\
(9.20-15.70)\end{array}$ & $\begin{array}{c}12.13 \pm 1.06 \\
(9.80-14.60)\end{array}$ & $\begin{array}{c}12.13 \pm 1.11 \\
(9.20-15.70)\end{array}$ & 0.990 \\
\hline $\begin{array}{l}\text { Postoperative hemoglobin level: mean } \pm \text { SD } \\
\quad \text { (minimum - maximum) }\end{array}$ & $\begin{array}{l}10.22 \pm 1.43 \\
(6.20-14.10)\end{array}$ & $\begin{array}{l}10.19 \pm 1.46 \\
(6.40-13.90)\end{array}$ & $\begin{array}{c}10.24 \pm 1.42 \\
(6.20-14.10)\end{array}$ & 0.785 \\
\hline
\end{tabular}

Abbreviation: SD—standard deviation. ${ }^{\star}$ Student's $\mathrm{t}$ test for independent samples. Significance set at $5 \%$ for all analys.

$t$-test, $\mathrm{p}>0.05$ for all), and the mean $( \pm \mathrm{SD})$ of preoperative and postoperative hematocrit and hemoglobin levels in immediate and delayed umbilical cord clamping were reduced in the 48 -h postoperative period (Student's paired $t$-test, $\mathrm{p} \leq 0.05$ for all, data not shown).

Among all maternal variables, only the oxytocin units used had a significant 
effect (Cohen's $\mathrm{d}=0.33 ; 95 \% \mathrm{CI}=-0.03-0.69)$. The others had small effect sizes (Cohen's d, $\Phi$ or $\Phi c \sim 0.1$ ) (data not shown).

Spearman's correlation analysis (Table 4$)$ revealed that the use of forceps $\left(r_{S}=\right.$ $0.109, \mathrm{p}=0.039)$ and an additional oxytocin dose $\left(\mathrm{r}_{\mathrm{S}}=0.125, \mathrm{p}=0.018\right)$ was positively related to delayed umbilical cord clamping. An additional oxytocin dose was negatively related to preoperative hematocrit level $\left(r_{s}=-0.106, p=0.045\right)$ and to 48 -h postoperative hematocrit $\left(\mathrm{r}_{\mathrm{S}}=-0.195, \mathrm{p} \leq 0.0001\right)$ and hemoglobin $\left(r_{S}=-0.211, p \leq 0.0001\right)$. Episiotomy, forceps, and the number of used uterotonics were also negatively related to 48 - $h$ postoperative hematocrit $\left(r_{S}=-0.216\right.$, $\mathrm{p} \leq 0.0001 ; \mathrm{r}_{\mathrm{S}}=-0.208, \mathrm{p} \leq 0.0001 ;$ and $\mathrm{r}_{\mathrm{S}}=-0.225, \mathrm{p} \leq 0.0001$, respectively) and hemoglobin levels $\left(\mathrm{r}_{\mathrm{S}}=-0.213, \mathrm{p} \leq 0.0001 ; \mathrm{r}_{\mathrm{S}}=-0.221, \mathrm{p} \leq 0.0001 ;\right.$ and $\mathrm{r}_{\mathrm{S}}=$ $-0.239, \mathrm{p} \leq 0.0001$, respectively). Besides, 48-h hematocrit and hemoglobin levels were negatively related to maternal severe bleeding $\left(\mathrm{r}_{\mathrm{S}}=-0.140, \mathrm{p}=0.08\right.$; and $r_{S}=-0.163, p=0.002$, respectively) and uterine atony $\left(r_{S}=-0.170, p=\right.$ 0.001 ; and $\mathrm{r}_{\mathrm{S}}=-0.189, \mathrm{p} \leq 0.0001$, respectively), while it was positively related to laceration $\left(r_{S}=0.157, p=0.003\right.$; and $r_{S}=0.145, p=0.006$, respectively $)$ and maternal BMI $\left(\mathrm{r}_{\mathrm{S}}=0.143, \mathrm{p} \leq 0.0001\right.$; and $\mathrm{r}_{\mathrm{S}}=-0.132, \mathrm{p}=0.013$, respectively) (Figure 1).

Table 4. Spearman's correlation between variables of interest.

\begin{tabular}{|c|c|c|c|c|c|c|c|c|c|c|}
\hline \multirow[t]{2}{*}{ Variable } & \multicolumn{2}{|c|}{$\begin{array}{l}\text { Delayed umbilical } \\
\text { cord clamping }\end{array}$} & \multicolumn{2}{|c|}{$\begin{array}{c}\text { Preoperative } \\
\text { hematocrit level }\end{array}$} & \multicolumn{2}{|c|}{$\begin{array}{c}\text { Preoperative } \\
\text { hemoglobin level }\end{array}$} & \multicolumn{2}{|c|}{$\begin{array}{c}\text { Postoperative } \\
\text { hematocrit level }\end{array}$} & \multicolumn{2}{|c|}{$\begin{array}{c}\text { Postoperative } \\
\text { hemoglobin level }\end{array}$} \\
\hline & rs & $\mathrm{P}^{*}$ & $\mathrm{rs}_{\mathrm{s}}$ & $\mathrm{P}^{\star}$ & rs & $\mathrm{P}^{\star}$ & rs & $\mathrm{P}^{*}$ & rs & $\mathrm{P}^{*}$ \\
\hline Maternal weight (kg) & 0.010 & 0.852 & 0.003 & 0.959 & 0.027 & 0.609 & 0.142 & 0.007 & 0.132 & 0.013 \\
\hline Maternal BMI $\left(\mathrm{kg} / \mathrm{m}^{2}\right)$ & 0.078 & 0.140 & 0.026 & 0.630 & 0.055 & 0.302 & 0.143 & 0.007 & 0.132 & 0.013 \\
\hline Gestational age (weeks) & 0.0026 & 0.629 & 0.047 & 0.381 & 0.018 & 0.733 & -0.073 & 0.171 & -0.072 & 0.178 \\
\hline Cesarian section delivery & 0.031 & 0.561 & 0.009 & 0.859 & 0.014 & 0.793 & -0.088 & 0.098 & -0.082 & 0.121 \\
\hline Episiotomy & -0.019 & 0.717 & 0.093 & 0.079 & 0.107 & 0.044 & -0.216 & $\leq 0.0001$ & -0.213 & $\leq 0.0001$ \\
\hline Forceps & 0.109 & 0.039 & 0.048 & 0.371 & 0.030 & 0.571 & -0.208 & $\leq 0.0001$ & -0.221 & $\leq 0.0001$ \\
\hline Oxytocin use & 0.100 & 0.060 & -0.126 & 0.017 & -0.110 & 0.038 & -0.227 & $\leq 0.0001$ & -0.241 & $\leq 0.0001$ \\
\hline Additional oxytocin dose & 0.125 & 0.018 & -0.106 & 0.045 & -0.094 & 0.076 & -0.195 & $\leq 0.0001$ & -0.211 & $\leq 0.0001$ \\
\hline Misoprostol use & 0.015 & 0.778 & -0.115 & 0.030 & -0.114 & 0.032 & -0.189 & $\leq 0.0001$ & -0.197 & $\leq 0.0001$ \\
\hline Methylergometrine use & 0.056 & 0.293 & 0.009 & 0.859 & 0.048 & 0.368 & -0.086 & 0.106 & -0.098 & 0.066 \\
\hline Number of used uterotonic & 0.101 & 0.056 & -0.123 & 0.020 & -0.107 & 0.043 & -0.225 & $\leq 0.0001$ & -0.239 & $\leq 0.0001$ \\
\hline Laceration & -0.083 & 0.117 & -0.023 & 0.663 & 0.003 & 0.948 & 0.157 & 0.003 & 0.145 & 0.006 \\
\hline Uterine atony & 0.038 & 0.473 & -0.093 & 0.078 & -0.083 & 0.116 & -0170 & 0.001 & -0.189 & $\leq 0.0001$ \\
\hline Maternal severe bleeding & 0.005 & 0.929 & -0.008 & 0.879 & -0.014 & 0.800 & -0.140 & 0.008 & -0.163 & 0.002 \\
\hline Hospital discharge (days) & 0.045 & 0.399 & -0.087 & 0.103 & -0.087 & 0.130 & -0.064 & 0.227 & -0.068 & 0.198 \\
\hline Newborn weight (g) & 0.009 & 0.866 & -0.115 & 0.029 & -0.133 & 0.012 & -0.114 & 0.032 & -0.131 & 0.014 \\
\hline
\end{tabular}

Abbreviations: BMI_body mass index; NICU—neonatal intensive care unit; $\mathrm{r}_{\mathrm{s}}$-Spearman's rho coefficient; TTN-transient tachypnea of the newborn. ${ }^{*}$ Spearman’s correlation. Significance set at $5 \%$ for all analyses. 


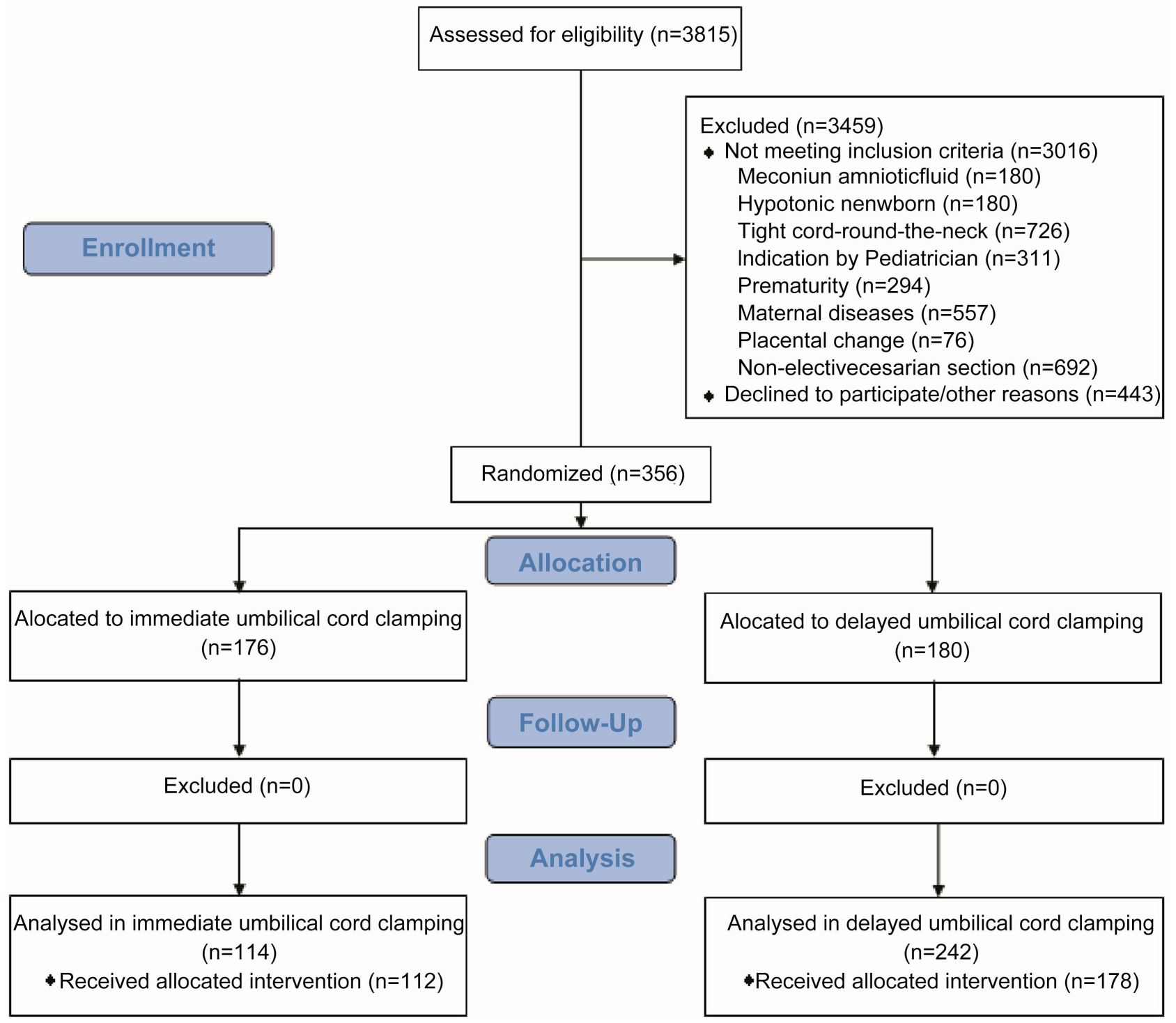

Figure 1. A flow chart for screening, enrollment, randomization, and follow-up of participants flow chart; $\mathrm{n}$-absolute frequency.

\section{Discussion}

\subsection{Principal Findings}

In this randomized clinical trial, delayed umbilical cord clamping ( $>60 \mathrm{~s})$ during vaginal and schedule cesarean term delivery did not alter maternal blood loss compared with immediate umbilical cord clamping. Indeed, in both umbilical cord clamping groups, the 48 -h postoperative hemoglobin and hematocrit levels were reduced to a similar extent. In the immediate umbilical cord clamping group, the mean $( \pm \mathrm{SD})$ difference between pre- and postoperative day 2 was 5.46 $\pm 3.88 \mathrm{~g} / \mathrm{dL}$ (hematocrit level) and $1.94 \pm 1.23 \mathrm{~g} / \mathrm{dL}$ (hemoglobin level). In the delayed umbilical cord group, the mean $( \pm S D)$ difference between pre- and postoperative day 2 was $5.19 \pm 3.93 \mathrm{~g} / \mathrm{dL}$ (hematocrit level) and $1.89 \pm 1.34 \mathrm{~g} / \mathrm{dL}$ (hemoglobin level).

The correlation analysis revealed that delayed umbilical cord clamping was 
positively related to the use of forceps and an additional oxytocin dose. Furthermore, an additional oxytocin dose was negatively related to preoperative hematocrit level and to the 48-h postoperative hematocrit level, while episiotomy, the use of forceps, and the number of used uterotonics were negatively related to the 48-h postoperative hematocrit and hemoglobin levels. In this sense, 48-h hematocrit and hemoglobin levels were negatively related to uterine atony and to severe maternal bleeding, and positively related to maternal BMI and to laceration.

Despite the differences between pre- and postoperative hematocrit and hemoglobin levels, there were no clinically important differences between the groups in maternal hemoglobin and hematocrit level and similar to scheduled full-term cesarean delivery [14] [15].

Medical concerns about the effects of delayed umbilical cord clamping during full-term vaginal or cesarean section deliveries may be a barrier to perform this practice. To our knowledge, this is the first randomized trial that has evaluated maternal outcomes with delayed cord clamping specifically in full-term vaginal and scheduled cesarean deliveries.

Considering maternal assessment, recently published studies examined the safety of delayed umbilical cord clamping after 60 and 90 - $120 \mathrm{~s}$ specifically for cesarean delivery [16] [17]. Similar to our results, these authors found no difference in maternal hemoglobin levels. Although the results from our study and Chantry et al. (2018) were less than the estimated results from Purisch et al. (2019), our results were not subjected to either subjectivity or temporary changes (e.g., use of historical controls) of the estimated blood loss assessment. Similar to our results, Purisch et al. (2019) also found no significant difference in maternal blood loss (e.g., postoperative hemoglobin levels) in women undergoing delayed umbilical cord clamping. In addition, previous studies have reported the effect of delayed umbilical cord clamping on possible slight falls or no effects in the umbilical cord blood $\mathrm{pH}$., with only one study showing a reduction in arterial $\mathrm{pH}$ and increased base excess by delayed umbilical cord clamping [18] [19] [20]. Although significant, this difference may be considered slight (with no clinical significance) and within acceptable physiological limits for both groups (immediate or delayed) in cesarean section at term deliveries, although further studies are recommended to better understand the potential risks of these practices for term newborns, considering the different birth routes [21] [22].

\subsection{Clinical Implications}

There were similar maternal outcomes for immediate and delayed umbilical cord clamping based on the actual intervention received rather than the intent to treat. Taken together, these findings indicate that medical teams should have additional uterotonics (e.g., oxytocin additional doses) to perform or manage, if necessary, umbilical cord clamping in, to minimize possible maternal and neonatal complications when there is early evidence of maternal moderate/severe bleeding. It is noteworthy that oxytocin is not free of side effects: It may cause hypotension, tachycardia, bradycardia, nausea and vomiting, hyponatremia and 
acute pulmonary edema and should be used with caution. This randomized controlled trial revealed that delayed umbilical cord clamping may be an acceptable alternative to immediate umbilical cord clamping because there were similar effects on maternal hematocrit and hemoglobin levels, and there were no increased risks for maternal complications.

\subsection{Research Implications}

We performed a study that compared immediate versus delayed umbilical cord clamping; hence, future research studies should be considered with a noninferiority design. In addition, our study was powered adequately to evaluate a difference in maternal hemoglobin and hematocrit levels, a surrogate measure for the transfusion need for the entire study population. However, additional studies that evaluate effects in mother with certain morbidities (e.g., anemia) are needed, because this group is at the highest risk for maternal blood loss and other maternal and neonatal adverse outcomes.

\section{Conclusion}

Among women undergoing low-risk vaginal or scheduled cesarean delivery of term singleton pregnancies, delayed umbilical cord clamping, compared with immediate umbilical cord clamping, resulted in a similar maternal hematocrit and hemoglobin level reduction at postoperative day 2 . In addition, there was not a clinically significant difference in delayed versus immediate umbilical cord clamping for maternal complications. In clinical practice, the implications of primary study outcomes should be considered based on the individual patient. Medical teams should have additional uterotonics to perform or manage, if necessary, umbilical cord clamping in the vaginal and cesarean sections at term deliveries, to minimize possible maternal complications.

\section{Authors' Contribution}

All authors listed above participated in the study to a significant extent. Gabriela Rostirolla, José Ramos, Edimárlei Valério, Charles Francisco Ferreira and Janete Vettorazzi worked on analysis and interpretation of the data. Gabriela Rostirolla, Maria Zanatta, Edimárlei Valério, and Janete Vettorazzi worked on the conception, design, and data collection. All authors read and approved the submitted manuscript.

\section{Funding}

The research was supported by the National Coordination for Improvement of Higher Education Personnel (CAPES, Brazil) and Research and Events Incentive Funding (FIPE/HCPA, Brazil).

\section{Conflicts of Interest}

None of the authors have any conflicts of interest related to this study, whether 
financial or of any other nature.

\section{References}

[1] Technical Working Group, World Health Organization (1997) Care in Normal Birth: A Practical Guide. Birth, 24, 121-123. https://doi.org/10.1111/j.1523-536X.1997.tb00352.x

[2] World Health Organization (2018) WHO Recommendations: Uterotonics for the Prevention of Postpartum Hamorrgage. https://apps.who.int/iris/bitstream/handle/10665/277276/9789241550420-eng.pdf?u $\underline{\mathrm{a}=1}$

[3] Elbourne, D.R., Prendiville, W.J., Carroli, G., Wood, J. and McDonald, S. (2001) Prophylactic Use of Oxytocin in the Third Stage of Labour. The Cochrane Database of Systematic Reviews, No. 4, CD001808.

[4] Masuzawa, Y., Kataoka, Y., Fujii, K. and Inoue, S. (2018) Prophylactic Management of Postpartum Haemorrhage in the Third Stage of Labour: An Overview of Systematic Reviews. Systematic Reviews, 7, 156.

https://doi.org/10.1186/s13643-018-0817-3

[5] Pates, J.A. and Satin, A.J. (2005) Active Management of Labor. Obstetrics and Gynecology Clinics of North America, 32, 221-230.

https://doi.org/10.1016/j.ogc.2005.01.004

[6] Committee on Obstetric Practice (2017) Committee Opinion No. 684: Delayed Umbilical Cord Clamping after Birth. Obstetrics and Gynecology, 129, e5-e10. https://doi.org/10.1097/AOG.0000000000001860

[7] Rabe, H., Diaz-Rossello, J.L., Duley, L. and Dowswell, T. (2012) Effect of Timing of Umbilical Cord Clamping and Other Strategies to Influence Placental Transfusion at Preterm Birth on Maternal and Infant Outcomes. The Cochrane Database of Systematic Reviews, No. 8, CD003248. https://doi.org/10.1002/14651858.CD003248.pub3

[8] Rabe, H., Gyte, G.M., Díaz-Rossello, J.L. and Duley, L. (2019) Effect of Timing of Umbilical Cord Clamping and Other Strategies to Influence Placental Transfusion at Preterm Birth on Maternal and Infant Outcomes. The Cochrane Database of Systematic Reviews, No. 9, CD003248. https://doi.org/10.1002/14651858.CD003248.pub4

[9] Purisch, S.E., Ananth, C.V., Arditi, B., Mauney, L., Ajemian, B., Heiderich, A., et al. (2019) Effect of Delayed vs Immediate Umbilical Cord Clamping on Maternal Blood Loss in Term Cesarean Delivery: A Randomized Clinical Trial. JAMA, 322, 1869-1876. https://doi.org/10.1001/jama.2019.15995

[10] Begley, C.M., Gyte, G.M., Devane, D., McGuire, W., Weeks, A. and Biesty, L.M. (2019) Active versus Expectant Management for Women in the Third Stage of Labour. The Cochrane Database of Systematic Reviews, 8, CD007412. https://doi.org/10.1002/14651858.CD007412.pub5

[11] World Health Organization (2012) WHO Recommendations for the Prevention and Treatment of Postpartum Haemorrhage. https://apps.who.int/iris/bitstream/handle/10665/75411/9789241548502 eng.pdf?se quence $=1$

[12] Weeks, A. (2015) The Prevention and Treatment of Postpartum Haemorrhage: What Do We Know, and Where Do We Go to Next? BJOG: An International Journal of Obstetrics and Gynaecology, 122, 202-210. 
https://doi.org/10.1111/1471-0528.13098

[13] Abramson, J.H. (2004) WINPEPI (PEPI-for-Windows): Computer Programs for Epidemiologists. Epidemiologic Perspectives \& Innovations, 1, 6.

https://doi.org/10.1186/1742-5573-1-6

[14] McDonald, S.J., Middleton, P., Dowswell, T. and Morris, P.S. (2013) Effect of Timing of Umbilical Cord Clamping of Term Infants on Maternal and Neonatal Outcomes. The Cochrane Database of Systematic Reviews, No. 7, CD004074. https://doi.org/10.1002/14651858.CD004074.pub3

[15] McDonald, S.J., Middleton, P., Dowswell, T. and Morris, P.S. (2014) Effect of Timing of Umbilical Cord Clamping of Term Infants on Maternal and Neonatal Outcomes. Evidence-Based Child Health: A Cochrane Review Journal, 9, 303-397. https://doi.org/10.1002/ebch.1971

[16] Chantry, C.J., Blanton, A., Taché, V., Finta, L. and Tancredi, D. (2018) Delayed Cord Clamping during Elective Cesarean Deliveries: Results of a Pilot Safety Trial. Maternal Health, Neonatology and Perinatology, 4, 16. https://doi.org/10.1186/s40748-018-0083-3

[17] De Paco, C., Florido, J., Garrido, M.C., Prados, S. and Navarrete, L. (2011) Umbilical Cord Blood Acid-Base and Gas Analysis after Early versus Delayed Cord Clamping in Neonates at Term. Archives of Gynecology and Obstetrics, 283, 1011-1014. https://doi.org/10.1007/s00404-010-1516-Z

[18] Giovannini, N., Crippa, B.L., Denaro, E., Raffaeli, G., Cortesi, V., Consonni, D., et al. (2020) The Effect of Delayed Umbilical Cord Clamping on Cord Blood Gas Analysis in Vaginal and Caesarean-Delivered Term Newborns without Fetal Distress: A Prospective Observational Study. BJOG: An International Journal of Obstetrics and Gynaecology, 127, 405-413. https://doi.org/10.1111/1471-0528.16026

[19] Rhoades, J.S., Wesevich, V.G., Tuuli, M.G., Macones, G.A. and Cahill, A.G. (2019) Implementation and Outcomes of Universal Delayed Umbilical Cord Clamping at Term. American Journal of Perinatology, 36, 233-242. https://doi.org/10.1055/s-0038-1669908

[20] Tang, J., Fullarton, R., Samson, S.-L. and Chen, Y. (2019) Delayed Cord Clamping Does Not Affect Umbilical Cord Blood Gas Analysis. Archives of Gynecology and Obstetrics, 299, 719-724. https://doi.org/10.1007/s00404-019-05048-5

[21] Valero, J., Desantes, D., Perales-Puchalt, A., Rubio, J., Diago Almela, V.J. and Perales, A. (2012) Effect of Delayed Umbilical Cord Clamping on Blood Gas Analysis. European Journal of Obstetrics, Gynecology, and Reproductive Biology, 162, 21-23. https://doi.org/10.1016/j.ejogrb.2012.01.020

[22] Wiberg, N., Källén, K. and Olofsson, P. (2008) Delayed Umbilical Cord Clamping at Birth Has Effects on Arterial and Venous Blood Gases and Lactate Concentrations. BJOG: An International Journal of Obstetrics and Gynaecology, 115, 697-703. https://doi.org/10.1111/j.1471-0528.2008.01708.x 